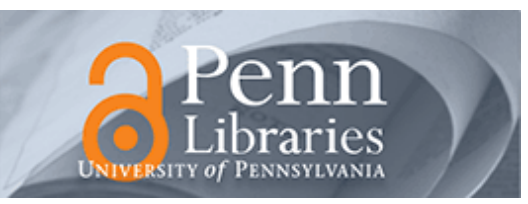

University of Pennsylvania

ScholarlyCommons

June 2003

\title{
Metamaterials with Negative Permittivity and Permeability: Background, Salient Features, and New Trends
}

\author{
Nader Engheta \\ University of Pennsylvania, engheta@seas.upenn.edu
}

Follow this and additional works at: https://repository.upenn.edu/ese_papers

\section{Recommended Citation}

Nader Engheta, "Metamaterials with Negative Permittivity and Permeability: Background, Salient Features, and New Trends", . June 2003.

Copyright 2003 IEEE. Reprinted from IEEE MTT-S International Microwave Symposium Digest 2003 Volume 1, pages 187-190.

Publisher URL: http://ieeexplore.ieee.org/xpl/tocresult.jsp?isNumber=27238\&page=2

This material is posted here with permission of the IEEE. Such permission of the IEEE does not in any way imply IEEE endorsement of any of the University of Pennsylvania's products or services. Internal or personal use of this material is permitted. However, permission to reprint/republish this material for advertising or promotional purposes or for creating new collective works for resale or redistribution must be obtained from the IEEE by writing to pubs-permissions@ieee.org. By choosing to view this document, you agree to all provisions of the copyright laws protecting it.

This paper is posted at ScholarlyCommons. https://repository.upenn.edu/ese_papers/9

For more information, please contact repository@pobox.upenn.edu. 


\title{
Metamaterials with Negative Permittivity and Permeability: Background, Salient Features, and New Trends
}

\author{
Abstract \\ Here we first present a brief background and the history of complex media, in particular the materials with \\ negative permittivity and permeability, and then we discuss some of the salient electromagnetic features \\ of these metamaterials. This is followed by description of some of the ideas regarding potential future \\ applications of these metamaterials in devices and components, along with physical remarks and \\ intuitive justification.

\section{Comments} \\ Copyright 2003 IEEE. Reprinted from IEEE MTT-S International Microwave Symposium Digest 2003 \\ Volume 1, pages 187-190. \\ Publisher URL: http://ieeexplore.iee. org/xpl/tocresult.jsp?isNumber=27238\&page=2 \\ This material is posted here with permission of the IEEE. Such permission of the IEEE does not in any way \\ imply IEEE endorsement of any of the University of Pennsylvania's products or services. Internal or \\ personal use of this material is permitted. However, permission to reprint/republish this material for \\ advertising or promotional purposes or for creating new collective works for resale or redistribution must \\ be obtained from the IEEE by writing to pubs-permissions@ieee.org. By choosing to view this document, \\ you agree to all provisions of the copyright laws protecting it.
}




\title{
Invited - Metamaterials with Negative Permittivity and Permeability: Background, Salient Features, and New Trends
}

\author{
Nader Engheta \\ University of Pennsylvania, Department of Electrical and Systems Engineering, \\ Philadelphia, Pennsylvania 19104-6390, U.S.A. \\ E-mail: engheta@ee.upenn.edu \\ URL: http://www.ee.upenn.edu/ engheta/
}

\begin{abstract}
Here we first present a brief background and the history of complex media, in particular the materials with negative permittivity and permeability, and then we discuss some of the salient electromagnetic features of these metamaterials. This is followed by description of some of the ideas regarding potential future applications of these metamaterials in devices and components, along with physical remarks and intuitive justification.
\end{abstract}

\section{INTRODUCTION}

The first attempt to explore the concept of "artificial" materials appears to begin in the late part of nineteenth century when in 1898 Jagadis Chunder Bose conducted the first microwave experiment on twisted structures, which by today's definition, was an artificial chiral "medium" [1]. Later in 1914, Lindman worked on "artificial" chiral media, which he formed by dispersing many randomly-oriented small wire helices in a host medium [2]. In 1948, Kock [3] made lightweight microwave lenses by arranging conducting spheres, disks, and strips periodically, and he demonstrated the possibility of tailoring the effective refractive index of the "artificial" media. Since then, the artificial complex materials have been the subject of research for many investigators worldwide. Such interest has been motivated by many aspects among which is the general idea that some of the devices and components that are made from naturally available optical materials can be constructed using "artificial" materials for applications in the lower frequencies. This can be achieved by length scaling [4]. Furthermore, and more importantly, in recent years new concepts in synthesis and novel fabrication techniques may allow construction of new classes of composite materials with interesting electromagnetic properties not easily available in nature. These metamaterials, which can in principle be synthesized by embedding various constituents/inclusions with novel geometrical shapes and forms in some host media (Fig. 1), possess exciting electromagnetic properties and response functions, not easily available or probable in nature but physically realizable, with new applications in the design of devices and components. Various types of electromagnetic composite media, such as chiral materials, omega media, wire media, bianisotropic media, linear and nonlinear media, and local and nonlocal media to name a few, have been studied by various research groups worldwide.

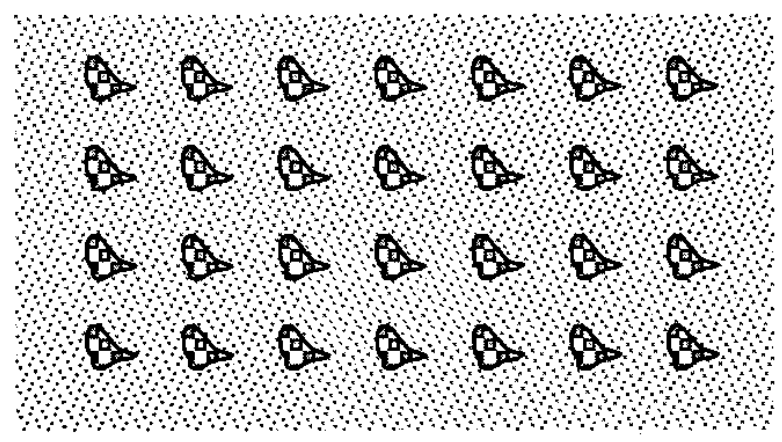

Fig. 1. A generic sketch for a metamaterial synthesized by embedding various inclusions in a host medium.

As is well known, in particulate composite media, electromagnetic waves interact with the inclusions, inducing electric and magnetic moments, which in turn affect the macroscopic effective permittivity and permeability of the bulk composite "medium". Since metamaterials can be synthesized by embedding artificially fabricated inclusions in a specified host medium or on a host surface, this provides the designer with a large collections of independent parameters (or degrees of freedom), such as the properties of host materials, the size, shape, and composition of the inclusions, and the density, arrangement, and alignment of these inclusions, to work with in order to engineer a metamaterial with a specific electromagnetic response functions not found in each of the individual constituent 
materials. Each and every one of these design parameters can play a key role in the final outcome of the synthesis and process. Among these, the geometry (or shape) of the inclusions is one that can provide a variety of new possibilities for metamaterials processing.

Recently, the idea of complex materials in which both permittivity and permeability possess negative real values at certain frequencies has received considerable attention (see e.g., [5]-[25]). In 1967, Veselago theoretically investigated plane wave propagation in a material whose permittivity and permeability were assumed to be simultaneously negative [9]. His theoretical study showed that for a monochromatic uniform plane wave in such a medium the direction of the Poynting vector is antiparallel to the direction of phase velocity, contrary to the case of plane wave propagation in conventional simple media. Recently, Smith et al. constructed such a composite medium for the microwave regime, and demonstrated experimentally the presence of anomalous refraction in this medium [5], [8]. For metamaterials with negative permittivity and permeability, several names and terminologies have been suggested, such as "left-handed" media [5], [6], [8], [9], [13], [15], media with negative refractive index [5], [6], [8], [9], [14], "backward wave media" (BW media) [10], "double negative (DNG)" metamaterials [11], to name a few.

Many research groups all over the world are now studying various aspects of this class of metamaterials, and several ideas and suggestions for future applications of these materials have been proposed. As for methods of constructions, several geometries for the inclusions of such media have been suggested. Among those, one can mention the thin wire and the split ring resonators (SRR) used originally by Smith, Schultz and Shelby [5], [8], inspired by the work of Pendry [7], capacitively loaded strips (CLS) and SRR by Ziolkowski [12], and the theory and numerical study for omega inclusions by Engheta, Nelatury, and Hoorfar [21].

\section{OMEGA MEDIUM AS A DNG MEDIUM}

In the early 1990's, Saadoun and Engheta theoretically introduced the idea of "omega" medium as a particulate medium conceptually made of many small inclusions in the shape of Greek letter $\Omega$ embedded in a host medium [25], and then in their theoretical work on analysis of wave propagation in "omega" media (both "local" and "nonlocal" omega media) they studied the modelling of effective permittivity, effective permeability and effective (omega) coupling coefficient in such media using the circuit-model approach for the omega inclusions [25]. Although not of interest to them at the time, their circuit-model analysis had also revealed the possibility of having negative permittivity and permeability in omega media for certain range of frequencies. In light of the recent interest in metamaterials with negative permittivity and permeability, Engheta, Nelatury and Hoorfar [21] have been exploring theoretically the possibility of extending Saadoun and Engheta's analysis. By studying electromagnetic wave interaction with the omega inclusions using the full-wave method-of-moment analysis, they evaluate frequency dependence of electric and magnetic polarizability tensors for omega inclusions, and then they obtain effective permittivity and effective permeability of bulk omega media using combined numerical and analytical techniques, with the goal of exploring situations when the omega media may possess negative permittivity and permeability at certain band of frequencies [21]. The theoretical analysis in this work has shown that, under certain conditions, omega media may indeed behave as a metamaterial with negative permittivity and permeability at some frequency band. One of the interesting features of the omega inclusion is that when it is in resonance, both the induced electric and magnetic dipole moments exhibit the resonant behaviour at the same frequency. This can lead to relative ease of design of appropriate omega structures for a desired range of frequency.

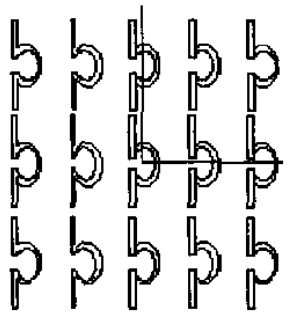

Fig. 2. Sketch of an omega medium.

\section{SUBWAVELENGTH DNG-DPS CAVITY AND WAVEGUIDE}

Exploiting the fact that the Poynting vector of a plane wave in a DNG medium is antiparallel with its phase velocity, and the corresponding anomalous refraction of a wave at the boundary between a DNG medium and a conventional medium (which can be called a "doublepositive (DPS)" medium), can lead to interesting ideas for novel devices and components. In one of our recent works in this area, we theoretically introduced the idea of thin subwavelength cavity resonators in which a pair of DPS and DNG layers was inserted ([17]-[19]). In that work, our theoretical results revealed that a slab of DNG 
metamaterial can act as a phase compensator/conjugator, and by pairing a DNG slab with a DPS slab one can, in principle, have a 1-D cavity resonator whose dispersion relation does not depend on the sum of thicknesses of the interior materials filling this cavity, but instead it depends on the ratio of these thicknesses [17], [19].

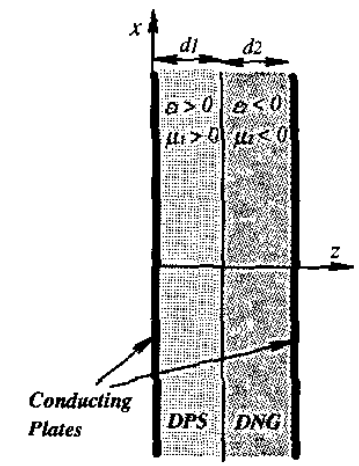

Fig. 3. An idea for a compact, thin, sub-wavelength cavity resonator or a waveguide. A pair of DPS and DNG slabs is placed between the two perfectly conducting plates. With the proper choice of ratio of $d_{l}$ over $d_{2}$, one can have a resonant cavity in which the ratio of $d_{1}$ and $d_{2}$ is the main constraint, not the sum of thicknesses, $d_{1}+d_{2}$. (see [17], [19] for more details.)

More explicitly, we have found that the dispersion characteristics of this 1-D cavity resonator can be written as

$\frac{\sqrt{\mu_{2} \varepsilon_{2}}}{\mu_{2}} \tan \left(\omega \sqrt{\mu_{1} \varepsilon_{1}} d_{1}\right)+\frac{\sqrt{\mu_{1} \varepsilon_{1}}}{\mu_{1}} \tan \left(\omega \sqrt{\mu_{2} \varepsilon_{2}} d_{2}\right)=0$

where the quantities $\mu_{1}, \varepsilon_{1}, \mu_{2}$, and $\varepsilon_{2}$ are generally all frequency dependent. It is important to note that the choice of sign for the square roots does not affect this dispersion relation. Either choice of sign (positive or negative sign) for the two square roots will leave this dispersion relation unchanged. Since the first layer is assumed to be made of a lossless DPS material, and the second layer is taken to be a lossless DNG metamaterial, we can write $\mu_{1}=\left|\mu_{1}\right|, \quad \varepsilon_{1}=\left|\varepsilon_{1}\right|, \quad \mu_{2}=-\left|\mu_{2}\right|$, and $\varepsilon_{2}=-\left|\varepsilon_{2}\right|$. As we have shown in [17], substituting these expressions in Eq. (1), we get

$$
\frac{\tan \left(\omega \sqrt{\left|\mu_{1} \varepsilon_{1}\right|} d_{1}\right)}{\tan \left(\omega \sqrt{\left|\mu_{2} \varepsilon_{2}\right|} d_{2}\right)}=\frac{\left|\mu_{2}\right| \sqrt{\left|\mu_{1} \varepsilon_{1}\right|}}{\left|\mu_{1}\right| \sqrt{\left|\mu_{2} \varepsilon_{2}\right|}}
$$

which shows no constraint on the sum of thicknesses of $d_{1}$ and $d_{2}$, and instead it only shows dependence on the ratio of tangent of these thicknesses (with multiplicative constants). So, in principle, $d_{1}$ and $d_{2}$ can conceptually be as thin or as thick as needed as long as the above ratio is satisfied. If we assume that $\omega, d_{1}$ and $d_{2}$ are chosen such that the small-argument approximation can be used for the tangent function, the above relation can be simplified as $\frac{d_{1}}{d_{2}} \cong \frac{\left|\mu_{2}\right|}{\left|\mu_{1}\right|}$, from which we can see that $d_{2} / d_{1}$ must satisfy the specific ratio in order to have a resonant mode with frequency $\omega$ in this cavity. Therefore, in principle, we can have a thin, subwavelength cavity resonator for a given frequency, if at this frequency the second layer acts as a DNG metamaterial, and the ratio, $d_{1} / d_{2}$, satisfies the above conditions.

We also analyzed the modes inside the parallel-plate waveguides containing a pair of parallel DPS and DNG layers (Fig. 3), for which the dispersion relations have the following form

$$
\frac{\tan \left(\sqrt{\omega^{2} \mu_{1} \varepsilon_{1}-\beta^{2}} d_{1}\right)}{\tan \left(\sqrt{\omega^{2} \mu_{2} \varepsilon_{2}-\beta^{2}} d_{2}\right)}=\frac{\sqrt{\omega^{2} \mu_{1} \varepsilon_{1}-\beta^{2}}\left|\mu_{2}\right|}{\sqrt{\omega^{2} \mu_{2} \varepsilon_{2}-\beta^{2}}\left|\mu_{1}\right|}
$$

where $\beta$ is the longitudinal wave number of the guided modes [18]. Again, we note that this dispersion relation does not constrain the total thickness of the waveguide $d_{1}+d_{2}$. Rather it includes the ratios of tangent of these quantities. Alu and Engheta have also investigated the modes in open DNG slab waveguides, and mode coupling between open DNG(and DPS slab waveguides [19], [20]. In each of these problems, we have found that when a DNG layer is combined with, or is in proximity of, a DPS layer interesting and unusual properties are observed for wave propagation within this structure. The paired DNGDPS bilayer structures exhibit even more interesting properties than a single DNG or DPS slab. These properties are specific to the wave interaction between the DNG and DPS layers. Such sub-wavelength cavity and guided-wave structures with no cut-off thicknesses, which may support resonant and propagating modes even at thicknesses much smaller than the wavelength, may provide possibilities for design of future miniaturized devices. We have also analyzed the modal structures in parallel-plate cavities and waveguides filled with a pair of conjugate "single-negative" layers, and have obtained their salient features [22].

\section{CONCLUSION}

Following a short review of the history of complex media and metamaterials, some of the salient features and potential ideas for metamaterial with negative refractive index have been discussed. It is shown that double 
negative media possess interesting features and electromagnetic response functions that can offer exciting possibilities for the future design of devices and components involving sub-wavelength structures. Some of the salient properties of these structures have been reviewed.

\section{REFERENCES}

[1] J. C. Bose, "On the Rotation of Plane of Polarisation of Electric Waves by a Twisted Structure," Proceedings of the Royal Society, Vol. 63, pp. 146-152, 1898.

[2] I. V. Lindell, A. H. Sihvola, and J. Kurkijarvi, "Karl F. Lindman: The Last Hertzian, and a Harbinger of Electromagnetic Chirality," IEEE Antennas and Propagation Magazine, vol. 34, No. 3, pp. 24-30, 1992.

[3] W. E. Kock, "Metallic Delay Lenses" Bell Systems Technical Journal, Vol. 27, pp. 58-82, 1948.

[4] A. F. Harvey, "Optical Techniques at Microwave Frequencies," Proc. of the IEE, Vol. 106,, Part B, pp. 141$157,1959$.

[5] D. R. Smith, W. J. Padilla, D. C. Vier, S. C. NematNasser, and S. Schultz, "Composite medium with simultaneously negative permeability and permittivity," Phys. Rev. Lett., vol. 84, no. 18, pp. 4184-4187, 1 May 2000.

[6] J. B. Pendry, "Negative refraction makes a perfect lens," Phys. Rev. Lett., vol. 85, no. 18, pp. 3966-3969, Oct 2000.

[7] J. B. Pendry, A. J. Holden, D. J. Robbins, W. J. Stewart, "Magnetism from conductors and enhanced nonlinear phenomena," IEEE Transactions on Microwave Theory and Techniques, vol. 47, No. 11, pp. 2075-2081, 1999.

[8] R. A. Shelby, D. R. Smith, S. Schultz, "Experimental verification of a negative index of refraction," Science, vol. 292, no. 5514, pp. 77-79, 6 April 2001.

[9] V. G. Veselago, "The electrodynamics of substances with simultaneously negative values of $\varepsilon$ and $\mu$," Soviet Physics Uspekhi, vol. 10, no. 4, pp. 509-514, 1968. [Usp. Fiz. Nauk, vol. 92, pp. 517-526, 1967.]

[10] I. V. Lindell, S. A. Tretyakov, K. I. Nikoskinen, and S. Ilvonen, "BW media - Media with negative parameters, capable of supporting backward waves," Microwave and Opt. Tech. Lett., Vol. 31, No. 2, pp. 129-133, Oct 2001.

[11] R. W. Ziolkowski, and E. Heyman, "Wave propagation in media having negative permittivity and permeability," Physical Review E., Vol. 64, No. 5, 056625, 2001.

[12] R. W. Ziolkowski, "Tailoring the electromagnetic responses of metamaterials: Double negative metamaterial designs and experiments," a talk at the IEEE-Nano' 2002 conference, Washington $D C$, August 26-28, 2002. Also see R.W. Ziolkowski, "Double Negative Metamaterial, Physics, Design and Experiments", Presented at KITP Quantum Optics, July 2002, available at http://online.kitp.ucsb.edu/online/qo02/ziolkowski/

[13] C. Caloz, C.-C. Chang, and T. Itoh, "Full-wave verification of the fundamental properties of left-handed materials in waveguide configurations," Journal of Applied Physics, Vol. 90, No. 11, pp. 5483-5486, December 2001.
[14] A. K. Iyer and G. V. Eleftheriades, "Negative Refractive Index Metamaterials Supporting 2-D Waves," 2002 IEEE MTT International Microwave Symposium (IMS), Seattle, WA, June 2-7, 2002, pp. 1067-1070 of Digest.

[15] C. Caloz, H. Okabe, T. Iwai, T. Itoh, "Transmission Line Approach of Left-Handed Materials", 2002 IEEE AP-S International Symposium and USNC/URSI National Radio Science Meeting, San Antonio (TX), June 16-21, 2002, URSI session, abstract on page 39.

[16] M. W. McCall, A. Lakhtakia, and W. S. Weiglhofer, "The negative index of refraction demystified," European Journal of Physics, vol. 23, pp. 353-359, 2002.

[17] N. Engheta, "An idea for thin subwavelength cavity resonators using metamaterials with negative permittivity and permeability," IEEE Antennas and Wireless Propagation Letters, Vol, 1, No. 1, 10-13, 2002.

[18] N. Engheta, "Guided Waves in Paired DielectricMetamaterial with Negative Permittivity and Permeability Layers" presented at the USNC-URSI National Radio Science Meeting, in Boulder, Colorado, January 9-12, 2002. Page 66.

[19] N. Engheta, "Ideas for potential applications of metamaterials with negative permittivity and permeability," a chapter in Advances in Electromagnetics of Complex Media and Metamaterials, NATO Science Series, (S. Zouhdi, A. H. Sihvola, M. Arsalane, editors), Kluwer Academic Publishers, pp. 19-37, 2002.

[20] A. Alù and N. Engheta, "Anomalous Mode Coupling in Guided-Wave Structures Containing Metamaterials with Negative Permittivity and Permeability," a talk at the IEEE-Nano' 2002 conference, Washington DC, Aug. 26-28, 2002.

[21] N. Engheta, S. Nelatury, and A. Hoorfar, "The Role of Geometry of Inclusions in Forming Metamaterials with Negative Permittivity and Permeability" a poster presented at the XXVII General Assembly of International Union of Radio Science (URSI GA'O2), in Maastricht, The Netherlands, August 17-24, 2002. Paper \# 1935 in the CD.

[22] A. Alù and N. Engheta, "Mono-modal waveguides filled with a pair of parallel epsilon-negative and mu-negative metamaterial layers," in the Digest of IEEE MTT Int. Microwave Symp. (IMS'03), Philadelphia, June 8-13, 2003.

[23] R. Marques, F. Medina, and R. Rafii-El-Idrissi, "Role of bianisotropy in genative permeability and left-handed metamaterials," Physical Review B, Vol. 65, No. 14, 144440, 2002.

[24] J. A. Kong, B.-I. Wu, and Y. Zhang, "A unique lateral displacement of a Gaussian beam transmitted through a slab with negative permittivity and permeability," Microwave and Opt. Tech. Lett., Vol. 33, No. 2, pp. 136$139,2002$.

[25] M. M. I. Saadoun and N. Engheta, "Theoretical Study of Electromagnetic Properties of Non-Local $\Omega$ Media" a chapter in Progress in Electromagnetics Research (PIER) Monograph Series, Vol. 9 on Bianisotropic and Bi-Isotropic Media and Applications, Alain Priou (ed.), December 1994, ch. 15, pp. 351-397. 